\title{
The Lawson number of a semitopological semilattice
}

\author{
Taras Banakh ${ }^{1,2} \cdot$ Serhii Bardyla ${ }^{3}$. Oleg Gutik ${ }^{1}$
}

Received: 20 November 2019 / Accepted: 15 February 2021 / Published online: 13 May 2021

(c) The Author(s) 2021

\begin{abstract}
For a Hausdorff topologized semilattice $X$ its Lawson number $\bar{\Lambda}(X)$ is the smallest cardinal $\kappa$ such that for any distinct points $x, y \in X$ there exists a family $\mathcal{U}$ of closed neighborhoods of $x$ in $X$ such that $|\mathcal{U}| \leq \kappa$ and $\bigcap \mathcal{U}$ is a subsemilattice of $X$ that does not contain $y$. It follows that $\bar{\Lambda}(X) \leq \bar{\psi}(X)$, where $\bar{\psi}(X)$ is the smallest cardinal $\kappa$ such that for any point $x \in X$ there exists a family $\mathcal{U}$ of closed neighborhoods of $x$ in $X$ such that $|\mathcal{U}| \leq \kappa$ and $\bigcap \mathcal{U}=\{x\}$. We prove that a compact Hausdorff semitopological semilattice $X$ is Lawson (i.e., has a base of the topology consisting of subsemilattices) if and only if $\bar{\Lambda}(X)=1$. Each Hausdorff topological semilattice $X$ has Lawson number $\bar{\Lambda}(X) \leq \omega$. On the other hand, for any infinite cardinal $\lambda$ we construct a Hausdorff zero-dimensional semitopological semilattice $X$ such that $|X|=\lambda$ and $\bar{\Lambda}(X)=\bar{\psi}(X)=\operatorname{cf}(\lambda)$. A topologized semilattice $X$ is called (i) $\omega$ Lawson if $\bar{\Lambda}(X) \leq \omega$; (ii) complete if each non-empty chain $C \subseteq X$ has inf $C \in \bar{C}$ and $\sup C \in \bar{C}$. We prove that for any complete subsemilattice $X$ of an $\omega$-Lawson semitopological semilattice $Y$, the partial order $\leq_{X}=\{(x, y) \in X \times X: x y=x\}$ of $X$ is closed in $Y \times Y$ and hence $X$ is closed in $Y$. This implies that for any continuous homomorphism $h: X \rightarrow Y$ from a complete topologized semilattice $X$ to an $\omega$ Lawson semitopological semilattice $Y$ the image $h(X)$ is closed in $Y$.
\end{abstract}

Communicated by Jimmie D. Lawson.

Serhii Bardyla was supported by the Austrian Science Fund FWF (Grant M 2967).

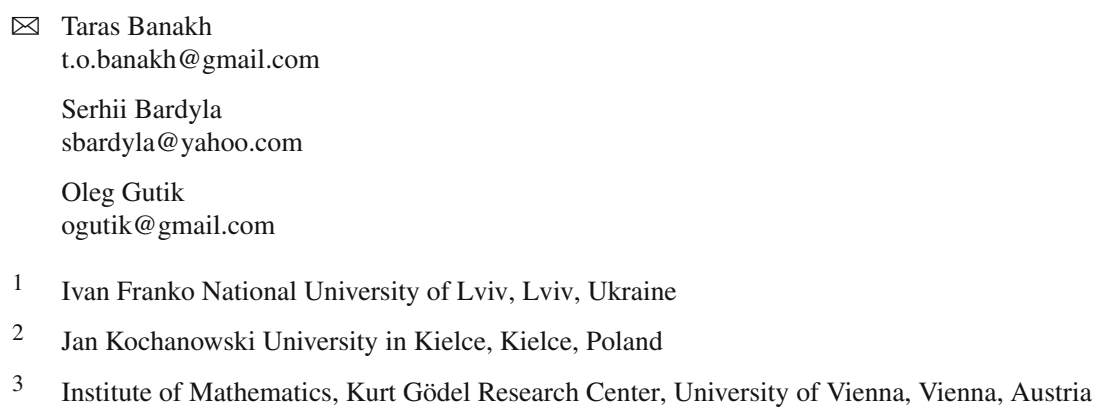


Keywords Semitopological semilattice $\cdot$ Complete topologized semilattice $\cdot$ Lawson number $\cdot \omega$-Lawson topologized semilattice

Mathematics Subject Classification 06B30 - 54D10

\section{Introduction}

In this paper we introduce a new cardinal invariant $\bar{\Lambda}(X)$ of a Hausdorff topologized semilattice $X$, called the Lawson number of $X$. This was motivated by studying the closedness properties of complete topologized semilattices, see [1-6]. It turns out that complete semitopological semilattices share many common properties with compact topological semilattices, in particular their continuous homomorphic images in Hausdorff topological semilattices are closed.

A semilattice is any commutative semigroup of idempotents (an element $x$ of a semigroup is called an idempotent if $x x=x$ ).

Each semilattice $X$ carries the natural partial order $\leq_{X}$ defined by $x \leq_{X} \quad y$ iff $x y=x=y x$. Many properties of semilattices are defined in the language of the natural partial order. In particular, for any point $x \in X$ we can consider its upper and lower sets

$$
\uparrow x:=\{y \in X: x y=x\} \text { and } \downarrow x:=\{y \in X: x y=y\}
$$

in the partially ordered set $\left(X, \leq_{X}\right)$.

A subset $C$ of a semilattice $X$ is called a chain if $x y \in\{x, y\}$ for any $x, y \in C$. A semilattice $X$ is called chain-finite if each chain in $X$ is finite. A semilattice is called linear if it is a chain in itself.

A semilattice endowed with a topology is called a topologized semilattice. A topologized semilattice $X$ is called a (semi )topological semilattice if the semigroup operation $X \times X \rightarrow X,(x, y) \mapsto x y$, is (separately) continuous.

In [16] Stepp proved that for any homomorphism $h: X \rightarrow Y$ from a chain-finite semilattice to a Hausdorff topological semilattice $Y$, the image $h(X)$ is closed in $Y$. In [1], the first authors improved this result of Stepp proving the following theorem.

Theorem 1 (Banakh, Bardyla) For any homomorphism $h: X \rightarrow Y$ from a chain-finite semilattice to a Hausdorff semitopological semilattice $Y$, the image $h[X]$ is closed in $Y$.

Topological generalizations of the notion of chain-finiteness are the notions of chain-compactness and completeness, discussed in [5].

A topologized semilattice $X$ is called

- chain-compact if each closed chain in $X$ is compact;

- complete if each non-empty chain $C \subseteq X$ has inf $C \in \bar{C}$ and $\sup C \in \bar{C}$.

Here $\bar{C}$ stands for the closure of $C$ in $X$. Chain-compact and complete topologized semilattices appeared to be very helpful in studying the closedness properties of topologized semilattices, see [1-6,11]. By Theorem 3.1 [1], a Hausdorff semitopological 
semilattice is chain-compact if and only if it is complete (see also Theorem 4.3 [5] for generalization of this characterization to topologized posets). In [1] the first two authors proved the following closedness property of complete topologized semilattices.

Theorem 2 (Banakh, Bardyla) For any continuous homomorphism $h: X \rightarrow Y$ from a complete topologized semilattice $X$ to a Hausdorff topological semilattice $Y$, the image $h[X]$ is closed in $Y$.

Theorems 1 and 2 motivate the following (still) open problem.

Problem 1 Assume that $h: X \rightarrow Y$ is a continuous homomorphism from a complete topologized semilattice $X$ to a Hausdorff semitopological semilattice $Y$. Is $h[X]$ closed in $Y$ ?

In [4] the first two authors gave the following partial answer to Problem 1.

Theorem 3 (Banakh, Bardyla) For any continuous homomorphism $h: X \rightarrow Y$ from a complete topologized semilattice $X$ to a sequential Hausdorff semitopological semilattice $Y$, the image $h[X]$ is closed in $Y$.

Another partial result to Problem 1 was given in [6].

Theorem 4 (Banakh, Bardyla, Ravsky) For any continuous homomorphism $h: X \rightarrow$ $Y$ from a complete topologized semilattice $X$ to a functionally Hausdorff semitopological semilattice $Y$, the image $h[X]$ is closed in $Y$.

Let us recall that a topological space $X$ is functionally Hausdorff if for any distinct points $x, y \in X$ there exists a continuous map $f: X \rightarrow \mathbb{R}$ such that $f(x) \neq f(y)$.

In fact, in [6] Theorem 4 was derived from the following closedness property of the partial order of a complete subsemilattice of a functionally Hausdorff semitopological semilattice.

Theorem 5 (Banakh, Bardyla, Ravsky) For any complete subsemilattice X of a functionally Hausdorff semitopological semilattice $Y$, the partial order $\leq_{X}$ of $X$ is a closed subset of $Y \times Y$.

In this paper we shall show that the answer to Problem 1 is affirmative under the additional condition that the semitopological semilattice $Y$ is $\omega$-Lawson. We shall also prove a counterpart of Theorem 5 for complete subsemilattices of $\omega$-Lawson semitopological semilattices.

We define a topologized semilattice $X$ to be $\omega$-Lawson if for any distinct points $x, y \in X$ there exists a countable family $\mathcal{U}$ of closed neighborhoods of $x$ such that $\bigcap \mathcal{U}$ is a subsemilattice of $X$ that does not contain $y$. A topologized semilattice $X$ is $\omega$-Lawson if and only if it is Hausdorff and has at most countable Lawson number $\bar{\Lambda}(X)$.

The Lawson number $\bar{\Lambda}(X)$ of a Hausdorff topologized semilattice $X$ is defined as the smallest cardinal $\kappa$ such that for any distinct points $x, y \in X$ there exists a family $\mathcal{U}$ of closed neighborhoods of $x$ such that $|\mathcal{U}| \leq \kappa$ and $\bigcap \mathcal{U}$ is a subsemilattice of $X$ that does not contain $y$. 
The Lawson number will be studied in more details in Sect. 2. In that section we shall prove that every Hausdorff topological semilattice $X$ has $\bar{\Lambda}(X) \leq \omega$. On the other hand, for any infinite cardinal $\lambda$ we shall construct a Hausdorff zero-dimensional semitopological semilattice $X$ of cardinality $|X|=\lambda$ and Lawson number $\bar{\Lambda}(X)=$ $\operatorname{cf}(\lambda)$. In Sect. 4 we prove the main result of this paper:

Theorem 6 For any complete subsemilattice $X$ of an $\omega$-Lawson semitopological semilattice $Y$, the natural partial order $\leq x$ of $X$ is a closed subset of $Y \times Y$. Consequently, $X$ is closed in $Y$.

Remark 1 By [7] (and [8]), there exists a metrizable (Lawson) semitopological semilattice $X$ whose partial order $\leq_{X}$ is not closed in $X \times X$.

Since the completeness is preserved by continuous homomorphisms into Hausdorff semitopological semilattices (see Lemma 1), Theorem 6 implies the following corollary giving a partial answer to Problem 1.

Corollary 1 For any continuous homomorphism $h: X \rightarrow Y$ from a complete topologized semilattice to an $\omega$-Lawson semitopological semilattice $Y$ the image $h[X]$ is closed in $Y$.

Problem 2 Let $X$ be a complete subsemilattice of an $\omega_{1}$-Lawson semitopological semilattice $Y$. Is $X$ closed in $Y$ ? Is the natural partial order $\leq_{X}$ of $X$ closed in $X \times X$ ?

\section{The Lawson number of a Hausdorff topologized semilattice}

In this section we study the Lawson number of a topologized semilattice in more details. We start with recalling the principal definition.

Definition 1 The Lawson number $\bar{\Lambda}(X)$ of a Hausdorff topologized semilattice $X$ is the smallest cardinal $\kappa$ such that for any distinct points $x, y \in X$ there exists a family $\mathcal{U}$ of closed neighborhoods of $x$ such that $|\mathcal{U}| \leq \kappa$ and the intersection $\bigcap \mathcal{U}$ is a subsemilattice of $X$ that does not contain $y$.

For any Hausdorff topologized semilattice $X$ the Lawson number $\bar{\Lambda}(X)$ is welldefined and does not exceed the closed pseudocharacter $\bar{\psi}(X)$ of $X$, defined as the smallest cardinal $\kappa$ such that for any point $x \in X$ there exists a family $\mathcal{U}$ of closed neighborhoods of $x$ such that $|\mathcal{U}| \leq \kappa$ and $\bigcap \mathcal{U}=\{x\}$. Therefore, $\bar{\Lambda}(X) \leq \bar{\psi}(X)$ for any Hausdorff topologized semilattice $X$.

Observe that a topologized semilattice $X$ is $\omega$-Lawson if and only if $X$ is Hausdorff and has $\bar{\Lambda}(X) \leq \omega$.

Definition 2 A topologized semilattice $X$ is defined to be $\kappa$-Lawson for a cardinal $\kappa$ if $X$ is Hausdorff and $\bar{\Lambda}(X) \leq \kappa$.

The Lawson number admits the following simple characterization.

Proposition 1 The Lawson number of a Hausdorff topologized semilattice $X$ is equal to the smallest cardinal $\kappa$ such that for any distinct points $x, y \in X$ there exist a closed subsemilattice $L$ in $X$ and a family $\mathcal{V}$ of closed neighborhoods of $x$ such that $|\mathcal{V}| \leq \kappa$ and $\bigcap \mathcal{V} \subseteq L \subseteq X \backslash\{y\}$. 
Proof We should prove that $\bar{\Lambda}(X)=\Lambda(X)$ where $\Lambda(X)$ is the smallest cardinal $\kappa$ such that for any distinct points $x, y \in X$ there exist a closed subsemilattice $L$ in $X$ and a family $\mathcal{V}$ of closed neighborhoods of $x$ such that $|\mathcal{V}| \leq \kappa$ and $\cap \mathcal{V} \subseteq L \subseteq X \backslash\{y\}$.

To see that $\Lambda(X) \leq \bar{\Lambda}(X)$, for any distinct points $x, y \in X$ use the definition of $\bar{\Lambda}(X)$ and find a family $\mathcal{U}$ of closed neighborhoods of $x$ such that $|\mathcal{U}| \leq \bar{\Lambda}(X)$ and $L:=\bigcap \mathcal{U}$ is a closed subsemilattice of $X$ that does not contain $y$. Then $\bigcap \mathcal{U}=L \subseteq$ $X \backslash\{y\}$, witnessing that $\Lambda(X) \leq \bar{\Lambda}(X)$.

To see that $\bar{\Lambda}(X) \leq \Lambda(X)$, for any distinct points $x, y \in X$ use the definition of $\Lambda(X)$ and find a closed subsemilattice $L$ of $X$ and a family $\mathcal{V}$ of closed neighborhoods of $x$ such that $|\mathcal{V}| \leq \Lambda(X)$ and $\bigcap \mathcal{V} \subseteq L \subseteq X \backslash\{y\}$. Then $\mathcal{U}:=\{V \cup L: V \in \mathcal{V}\}$ is a family of closed neighborhoods of $x$ such that $|\mathcal{U}| \leq|\mathcal{V}| \leq \Lambda(X)$ and $\bigcap \mathcal{U}=$ $(\bigcap \mathcal{V}) \cup L=L$ is a closed subsemilattice of $X$ that does not contain $y$ and witnesses that $\bar{\Lambda}(X) \leq \bar{\Lambda}(X)$.

The notion of a 1-Lawson semilattice extends the well-known notion of a Lawson semilattice (or else a topologized semilattice with small subsemilattices), introduced and studied by Lawson [13] (see also [10, Chapter 2]). Following [10, p. 12], we define a topologized semilattice $X$ to be

- Lawson if it has a base of the topology consisting of open subsemilattices;

- a $V$-semilattice if for any points $x \in X$ and $y \in X \backslash \uparrow x$ there exists a point $v \in X \backslash \downarrow y$ such that $\uparrow v$ is a neighborhood of $x$ in $X$;

- I-separated if for any distinct points $x, y \in X$ there exists a continuous homomorphism $f: X \rightarrow \mathbb{I}$ such that $f(x) \neq f(y)$.

Here by $\mathbb{I}$ we denote the unit interval $[0,1]$ endowed with the semilattice operation min.

Proposition 2 For a Hausdorff semitopological semilattice X, consider the following conditions:

1. X is 1-Lawson;

2. $X$ is Lawson;

3. $X$ is a $V$-semilattice;

4. $X$ is $\mathbb{I}$-separated.

Any of the conditions (2),(3),(4) implies (1). If the space $X$ is compact, then the conditions (1)-(4) are equivalent.

Proof To prove that $X$ is 1-Lawson, fix any distinct points $x, y \in X$. We need to find a closed subsemilattice $L$ in $X$ that contains $x$ in its interior but does not contain $y$.

(2) $\Rightarrow$ (1) Assume that $X$ is Lawson. Since $X$ is Hausdorff, there exists a closed neighborhood $N_{x} \subseteq X$ of $x$ such that $y \notin N_{x}$. Since $X$ is Lawson, there exists an open subsemilattice $V \subseteq X$ such that $x \in V \subseteq N_{x}$. Since $X$ is a semitopological semilattice, the closure $\overline{\bar{V}}$ of the semilattice $V$ is a closed subsemilattice that contains $x$ in its interior but does not contain $y$. Therefore, the semilattice $X$ is 1-Lawson.

(3) $\Rightarrow$ (1) Assume that $X$ is a $V$-semilattice. If $x \notin \downarrow y$, then $y \notin \uparrow x$ and there exists an element $v \in X \backslash \downarrow y$ such that the upper set $\uparrow v$ contains $x$ in its interior. Since $X$ is a Hausdorff semitopological semilattice the upper set $\uparrow v=\{z \in X: z v=v\}$ 
is closed. Then the closed subsemilattice $\uparrow v$ is a neighborhood of $x$ that does not contain $y$. If $x \in \downarrow y$, then $x \notin \uparrow y$. Since $X$ is a $V$-semilattice, there exists an element $u \in X \backslash \downarrow x$ such that the upper set $\uparrow u$ contains $y$ in its interior. Observe that the complement $U:=X \backslash \uparrow u$ is an open subsemilattice of $X$, containing $x$. Then $\bar{U}$ is a closed subsemilattice of $X$, which is a neighborhood of $x$ that does not contain $y$.

$(4) \Rightarrow$ (1) Assuming that $X$ is $\mathbb{I}$-separated, we can find a continuous homomorphism $f: X \rightarrow \mathbb{I}$ such that $f(x) \neq f(y)$. Choose any closed neighborhood $N \subset \mathbb{I}$ of $f(x)$ such that $f(y) \notin N$. Then $f^{-1}(N)$ is a closed subsemilattice in $X$ that contains $x$ in its interior but does not contain $y$.

Now assume that $X$ is compact. In this case the conditions (1)-(4) are equivalent by Theorem 7.1 in [2]. In fact, the equivalence of the conditions (2) and (4) is a classical result of Lawson $[13,14]$.

Example 1 The topological semilattice $\mathbb{Z}^{\omega}$ with the Tychonoff product topology and coordinatewise operation of minimum is Lawson and I-separated but not a $V$ semilattice.

By [10, Example 2.21], there exists a metrizable compact topological semilattice, which is not Lawson and hence is not 1-Lawson. However, such a semilattice necessarily is $\omega$-Lawson as shown by the following simple proposition.

Proposition 3 Each Hausdorff topological semilattice $X$ is $\omega$-Lawson.

Proof Given two distinct points $x, y \in X$, choose a decreasing sequence $\left(U_{n}\right)_{n \in \omega}$ of open neighborhoods of $x$ such that $y \notin \overline{U_{0}}$ and $U_{n} \cdot U_{n} \subseteq U_{n-1}$ and hence $\overline{U_{n}} \cdot \overline{U_{n}} \subseteq \overline{U_{n-1}}$ for all $n \in \mathbb{N}$. The choice of $U_{0}$ is possible by the Hausdorff property of $X$, and the choice of the neighborhoods $U_{n}$ is possible by the continuity of the semilattice operation at $(x, x)$. It follows that the intersection $\bigcap_{n \in \omega} \overline{U_{n}}$ is a closed subsemilattice of $X$ containing $x$ but not $y$.

Corollary 2 Each compact Hausdorff semitopological semilattice is $\omega$-Lawson.

Proof By [14], each compact Hausdorff semitopological semilattice is a topological semilattice and by Proposition 3, is $\omega$-Lawson.

Let us also notice the following trivial (but useful) fact.

Proposition 4 Each (Hausdorff) linear topologized semilattice X is Lawson (and 1Lawson).

Let us recall that a topological space $X$ is Urysohn if any distinct points in $X$ have disjoint closed neighborhoods. It is clear that each Urysohn space is Hausdorff.

We define a topological space $X$ to be $\kappa$-Urysohn for a cardinal $\kappa$ if for any distinct points $x, y \in X$ there are families $\mathcal{U}_{x}$ and $\mathcal{U}_{y}$ of open sets on $X$ such that $\max \left\{\left|\mathcal{U}_{x}\right|,\left|\mathcal{U}_{y}\right|\right\} \leq \kappa, x \in \bigcap \mathcal{U}_{x}, y \in \bigcap \mathcal{U}_{y}$ and the sets $\bigcap_{U \in \mathcal{U}_{x}} \bar{U}$ and $\bigcap_{V \in \mathcal{U}_{y}} \bar{V}$ are disjoint.

It is easy to see that a topological space is Urysohn if and only if it is 1-Urysohn.

Example 2 For every infinite cardinal $\kappa$, there exists a Hausdorff Lawson topological semilattice, which is not $\kappa$-Urysohn. 
Proof Take any ordinal $\lambda$ of cofinality $\operatorname{cf}(\lambda)>\kappa$ (for example, put $\lambda:=\kappa^{+}$). Consider the set $L=\left\{x_{\alpha}\right\}_{\alpha \leq \lambda} \cup\{z\} \cup\left\{y_{\alpha}\right\}_{\alpha \leq \lambda}$ of pairwise distinct points endowed with the linear order in which $x_{\alpha}<x_{\beta}<z<y_{\beta}<y_{\alpha}$ for any ordinals $\alpha<\beta \leq \lambda$. Let $\ddot{L}:=L \backslash\left\{x_{\lambda}, y_{\lambda}\right\}$. On the set

$$
X=(\ddot{L} \times[0, \lambda)) \cup\left(\left\{x_{\lambda}, y_{\lambda}\right\} \times\{\lambda\}\right)
$$

consider the semilattice operation

$$
(x, \alpha) \cdot(y, \beta):= \begin{cases}(\min \{x, y\}, \min \{\alpha, \beta\}) & \text { if } \alpha, \beta<\lambda ; \\ (\min \{x, z\}, \alpha), & \text { if } \alpha<\lambda=\beta ; \\ (\min \{z, y\}, \beta), & \text { if } \beta<\lambda=\alpha ; \\ (\min \{x, y\}, \lambda), & \text { if } \alpha=\lambda=\beta .\end{cases}
$$

Endow $X$ with the topology $\tau$ consisting of all sets $U \subseteq X$ satisfying the following three conditions:

- if $(z, \alpha) \in U$ for some $\alpha \in[0, \lambda)$, then $\left\{\left(x_{\gamma}, \alpha\right),\left(y_{\gamma}, \alpha\right): \beta<\gamma<\lambda\right\} \subseteq U$ for some $\beta \in[0, \lambda)$;

- if $\left(x_{\lambda}, \lambda\right) \in U$, then $\left\{\left(x_{\beta}, \gamma\right): \beta, \gamma \in[\alpha, \lambda)\right\} \subseteq U$ for some $\alpha \in[0, \lambda)$;

- if $\left(y_{\lambda}, \lambda\right) \in U$, then $\left\{\left(y_{\beta}, \gamma\right): \beta, \gamma \in[\alpha, \lambda)\right\} \subseteq U$ for some $\alpha \in[0, \lambda)$.

Taking into account that $\operatorname{cf}(\lambda)>\kappa$, we can show that $(X, \tau)$ is a required Hausdorff Lawson topological semilattice which is not $\kappa$-Urysohn.

Now we construct Hausdorff zero-dimensional semitopological semilattices having an arbitrarily large Lawson number. We recall that a topological space is zerodimensional if it has a base of the topology consisting of open-and-closed sets.

Example 3 For any infinite cardinal $\lambda$ there exists a Hausdorff zero-dimensional semitopological semilattice $X$ such that $|X|=\lambda$ and $\bar{\Lambda}(X)=\bar{\psi}(X)=\operatorname{cf}(\lambda)$.

Proof Consider the set

$$
X:=\{A \subseteq \lambda: A=\lambda \text { or } A \text { is finite }\}
$$

endowed with the semilattice operation of union. This semilattice has cardinality $|X|=\lambda$. Here we identify the cardinal $\lambda$ with the set $[0, \lambda)$ of all ordinals smaller than $\lambda$.

Now the trick is to introduce an appropriate topology on the semilattice $X$. For this we define several kinds of sets in $\lambda$.

A finite subset $A \subseteq \lambda$ is defined to be sparse if $|A \cap[\alpha, \alpha+\omega)| \leq 1$ for any ordinal $\alpha \in \lambda$.

For a set $A \in X$ and an ordinal $\alpha \in \lambda$ consider the set

$$
S[A ; \alpha]:=\{B \in X: B \cap[0, \alpha)=A \cap[0, \alpha) \text { and } B \cap[\alpha, \lambda) \text { is sparse }\},
$$

and observe that $\lambda \notin S[A ; \alpha]$. 
Let $\alpha \in \lambda$ be an ordinal, $n$ be a finite ordinal and $\varepsilon$ be a positive real number. A subset $A \subset \lambda$ is called $(\alpha, n, \varepsilon)$-fat if there exists a limsit ordinal $\beta \in[\alpha, \lambda)$ and a finite ordinal $m>n$ such that

(i) the set $A \cap[\beta+\omega, \lambda)$ is sparse;

(ii) $[\beta, \beta+\omega) \cap A=[\beta, \beta+m]$;

(iii) the set $[0, \beta) \cap A$ is finite and has cardinality $<\varepsilon \cdot m$.

The conditions (i),(ii) ensure that the ordinal $\beta$ is unique.

Consider the subset

$$
F[\alpha, n, \varepsilon]:=\{\lambda\} \cup\{A \in X: A \text { is }(\alpha, n, \varepsilon) \text {-fat }\}
$$

of $X$.

Now we define a topology $\tau$ on the semilattice $X$. This topology consists of the sets $U \subseteq X$ satisfying the following two conditions:

(a) for any finite subset $A \in U$ of $\lambda$ there exists an ordinal $\alpha \in \lambda$ such that $A \in$ $S[A, \alpha] \subseteq U$

(b) if $\lambda \in U$, then there exist ordinals $\alpha \in \lambda, k \in \omega$, and a positive real number $\varepsilon$ such that $F[\alpha, k, \varepsilon] \subseteq U$.

It is easy to see that $\tau$ is a well-defined topology on $X$. Now we show that this topology is Hausdorff and zero-dimensional. Subsets $U \in \tau$ of $X$ will be called $\tau$-open.

Claim 1 For any element $A \in X$ and ordinal $\alpha \in \lambda$ the set $S[A ; \alpha]$ is open in $(X, \tau)$.

Proof To see that $S[A ; \alpha]$ is open, take any element $B \in S[A ; \alpha]$ and observe that $B \cap[0, \alpha)=A \cap[0, \alpha)$ and $B \cap[\alpha, \lambda)$ is sparse. Then $B \in S[B ; \alpha] \subseteq S[A ; \alpha] \subseteq X \backslash\{\lambda\}$ and the set $S[A ; \alpha]$ is open by the definition of the topology $\tau$.

Claim 2 For any ordinals $\alpha \in \lambda, n \in \omega$ and a positive real number $\varepsilon$, the set $F[\alpha, n, \varepsilon]$ is open and closed in $(X, \tau)$.

Proof Given any element $A \in F[\alpha, n, \varepsilon] \backslash\{\lambda\}$, find a unique limit ordinal $\beta \geq \alpha$ witnessing that $A$ is $(\alpha, n, \varepsilon)$-fat. Then $S[A ; \beta+\omega] \subseteq F[\alpha, n, \varepsilon]$, witnessing that the set $F[\alpha, n, \varepsilon]$ is open in $(X, \tau)$.

To see that this set is closed in $(X, \tau)$, choose any set $A \in X \backslash F[\alpha, n, \varepsilon]$. It follows that $A$ is a finite subset of $\lambda$, which is not $(\alpha, n, \varepsilon)$-fat. Let $\beta$ be the smallest limit ordinal such that the intersection $A \cap[\beta+\omega, \lambda)$ is sparse. Let $m \in \omega$ be the smallest finite ordinal such that $A \cap[\beta, \beta+\omega) \subseteq[\beta, \beta+m]$. Since $A$ is not $(\alpha, n, \varepsilon)$-fat, one of the following conditions holds:

1. $\beta<\alpha$;

2. $m \leq n$;

3. $\beta \geq \alpha$ and $m>n$ but $[\beta, \beta+m] \nsubseteq A$;

4. $\beta \geq \alpha, m>n,[\beta, \beta+m] \subseteq A$ but $|A \cap[0, \beta)| \geq \varepsilon \cdot m$.

In all these cases $S[A ; \beta+\omega]$ is a $\tau$-open neighborhood of $A$ such that $S[A ; \beta+\omega] \cap$ $F[\alpha, n, \varepsilon]=\emptyset$. 
Claim 3 For any finite set $A \in X$ of $\lambda$ and any ordinal $\alpha \in \lambda$, the set $S[A ; \alpha]$ is closed in $(X, \tau)$.

Proof Take any element $B \in X \backslash S[A ; \alpha]$. If $B=\lambda$, then $F[\alpha, 1,1]$ is a neighborhood of $B$, disjoint with $S[A ; \alpha]$.

If $B \neq \lambda$, then $B \notin S[A ; \alpha]$ implies that either $B \cap[\alpha, \lambda)$ is not sparse or $B \cap[\alpha, \lambda)$ is sparse but $B \cap[0, \alpha) \neq A \cap[0, \alpha)$. In the latter case $S[B ; \alpha]$ is a $\tau$-open neighborhood of $B$, disjoint with $S[A ; \alpha]$. So, we assume that $B \cap[\alpha, \lambda)$ is not sparse. In this case we can choose any ordinal $\beta \in \lambda$ with $B \subset[0, \beta)$ and observe that $S[B, \beta]$ is a $\tau$-open neighborhood of $B$ such that $S[B ; \beta] \cap S[A ; \alpha]=\emptyset$.

Claim 4 The topology $\tau$ is Hausdorff.

Proof Take any distinct elements $A, B \in X$. If $A$ and $B$ are finite subsets of $X$, then we can find an ordinal $\alpha \in \lambda$ such that $A \cup B \subseteq[0, \alpha)$ and observe that $S[A ; \alpha]$ and $S[B ; \alpha]$ are disjoint $\tau$-open neighborhoods of the elements $A$ and $B$, respectively.

If $B=\lambda$, then $A$ is a finite set, contained in $[0, \alpha)$ for some ordinal $\alpha \in \lambda$. In this case $S[A ; \alpha]$ and $F[\alpha, 1,1]$ are disjoint $\tau$-open neighborhoods of $A$ and $B$, respectively,

The case $A=\lambda$ can be considered by analogy.

Claims $1-4$ show that the topology $\tau$ is Hausdorff and zero-dimensional.

Claim 5 The topologized semilattice $(X, \tau)$ is semitopological.

Proof Given any element $a \in X$, we should prove that the shift $s_{a}: X \rightarrow X$, $s_{a}: x \mapsto a x$, is continuous. If $a=\lambda$, then $s_{a}(X)=\{\lambda\}$ is a singleton, so the continuity of $s_{a}$ is trivial. So, we assume that $a$ is a finite subset of $\lambda$. To check the continuity of the shift $s_{a}$ at a point $x \in X$, fix any neighborhood $O_{a x} \in \tau$ of the point $a x=x \cup a$.

If $x \neq \lambda$, then $a x \neq \lambda$ and by the definition of the topology $\tau$, there exists an ordinal $\alpha \in \lambda$ such that $a x \in S[a x ; \alpha] \subseteq O_{a x}$. Replacing $\alpha$ by a larger ordinal, we can assume that $a x \subseteq[0, \alpha)$. Then $O_{x}:=S[x ; \alpha]$ is a $\tau$-open neighborhood of $x$ such that $s_{a}\left(O_{x}\right) \subseteq S[a x ; \alpha] \subseteq O_{a x}$.

If $x=\lambda$, then $a x=\lambda$ and by the definition of the topology $\tau$, there exist $\alpha \in \lambda$, $k \in \omega$ and $\varepsilon>0$ such that $F[\alpha, k, \varepsilon] \subseteq O_{a x}$. Replacing $\alpha$ by a larger ordinal, we can assume that $a \subset[0, \alpha)$. Replacing $k$ by a larger number, we can assume that $|a| \leq \frac{1}{2} \varepsilon k$. In this case $O_{x}:=F\left[\alpha, k, \frac{1}{2} \varepsilon\right]$ is a $\tau$-open neighborhood of $x=\lambda$ such that $s_{a}\left(O_{x}\right) \subseteq F[\alpha, k, \varepsilon] \subseteq O_{a x}$.

Claim 6 Let $\mathcal{U} \subseteq \tau$ be a family of open sets and $L$ be a $\tau$-closed subsemilattice in $X$ such that $|\mathcal{U}|<\operatorname{cf}(\lambda)$ and $\emptyset \neq \bigcap \mathcal{U} \subseteq L$. Then $\lambda \in L$.

Proof Fix any element $x \in \bigcap \mathcal{U}$. If $x=\lambda$, then $\lambda=x \in \bigcap \mathcal{U} \subseteq L$ and we are done. So, we assume that $x$ is a finite subset of $\lambda$. Since the set $L$ is $\tau$-closed, the inclusion $\lambda \in L$ will follow as soon as we show that each neighborhood $O_{\lambda} \in \tau$ of $\lambda$ meets the set $L$. By Claim 2, there exist ordinals $\alpha \in \lambda, k \in \omega$ and a positive real number $\varepsilon$ such that $F[\alpha, k, \varepsilon] \subseteq O_{\lambda}$. 
By Claim 1 and $|\mathcal{U}| \leq \kappa<\operatorname{cf}(\lambda)$, there exists a limit ordinal $\beta \in[\alpha, \lambda)$ such that $x \in[0, \beta)$ and $x \in S[x ; \beta] \subseteq \bigcap \mathcal{U} \subseteq L$. Choose a finite ordinal $n>k$ such that $|x|<\varepsilon n$. Observe that for every ordinal $\gamma \in[\beta, \beta+n]$ the set $x \cup\{\gamma\}$ belongs to the semilattice $L \supseteq S[x ; \beta]$. Since $L$ is a subsemilattice, the $(\beta, k, \varepsilon)$-fat set $x \cup[\beta, \beta+n]$ belongs to $L \cap F[\beta, k, \varepsilon] \subseteq L \cap O_{\lambda}$.

Claim 7 The semitopological semilattice $(X, \tau)$ has $\bar{\Lambda}(X, \tau)=\bar{\psi}(X, \tau)=\operatorname{cf}(\lambda)$.

Proof Claim 6 implies that $\operatorname{cf}(\lambda) \leq \bar{\Lambda}(X, \tau)$. Since $\bar{\Lambda}(X, \tau) \leq \bar{\psi}(X, \tau)$, it remains to prove that $\bar{\psi}(X, \tau) \leq \operatorname{cf}(\lambda)$.

Choose a cofinal subset $C \subseteq \lambda$ of cardinality $|C|=\operatorname{cf}(\lambda)$. To see that $\bar{\psi}(X, \tau) \leq$ $\operatorname{cf}(\lambda)$, take any $A \in X$. If $A$ is a finite subset of $\lambda$, then $A \subseteq[0, \alpha)$ for some ordinal $\alpha \in \lambda$. Then $\{A\}=\bigcap_{\alpha \leq \gamma \in C} S[A ; \gamma]$. If $A=\lambda$, then $\{A\}=\{\lambda\}=\bigcap_{\gamma \in C} F[\gamma ; 1,1]$. In both cases the singleton $\{A\}$ is the intersection of $\operatorname{cf}(\lambda)$ many closed neighborhoods of $A$, witnessing that $\bar{\psi}(X, \tau) \leq \operatorname{cf}(\lambda)$.

\section{Complete topologized semilattices}

In this section we recall some known properties and characterizations of complete topologized semilattices.

By a poset we understand a set endowed with a partial order. A topologized poset is a poset endowed with a topology. So, each topologized semilattice is a topologized poset.

A subset $D$ of a poset $(X, \leq)$ is called

- a chain if any elements $x, y \in D$ are comparable in the sense that $x \leq y$ or $y \leq x$;

- up-directed if for any $x, y \in D$ there exists $z \in D$ such that $x \leq z$ and $y \leq z$;

- down-directed if for any $x, y \in D$ there exists $z \in D$ such that $z \leq x$ and $z \leq y$.

It is clear that each chain in a poset is both up-directed and down-directed.

A topologized poset $X$ is defined to be

- up-complete if any nonempty up-directed subset $U \subseteq X$ has the least upper bound $\sup U \in \bar{U}$ in $X$;

- down-complete if any nonempty down-directed subset $D \subseteq X$ has the greatest lower bound inf $D \in \bar{D}$ in $X$.

The proof of the following classical characterization can be found in $[9,12,15]$ or $[5,2.2]$.

Proposition 5 For a topologized poset $X$ the following conditions are equivalent:

1. X is up-complete;

2. Each non-empty chain $C \subseteq X$ has the least upper bound $\sup C \in \bar{C}$ in $X$.

Proposition 5 implies the following useful characterization of completeness in topologized semilattices.

Corollary 3 A topologized semilattice $X$ is complete if and only if it is up-complete and down-complete. 
This corollary implies that each closed subsemilattice of a complete topologized semilattice has the smallest element.

A topologized semilattice $Y$ is called $\uparrow$-closed if for every $y \in Y$ the upper set $\uparrow y=\{x \in Y: x y=y\}$ is closed in $Y$. It is easy to see that each $T_{1}$ semitopological semilattice is $\uparrow$-closed.

The following lemma (that can be derived from Corollary 3) is proved in [[5], Lemma 5.3].

Lemma 1 Let $h: X \rightarrow Y$ be a continuous surjective homomorphism between topologized semilattices. If $X$ is complete and $Y$ is $\uparrow$-closed, then the topologized semilattice $Y$ is complete.

\section{Proof of Theorem 6 and Corollary 1}

The proof of Theorem 6 is based on the following lemma.

Lemma 2 Let $X$ be a complete subsemilattice of a semitopological semilattice $Y$. Let a pair $(x, y) \in Y \times Y$ belong to the closure of the natural partial order $\leq_{X}$ of $X$ in $Y \times Y$, and let $\left\{U_{n}\right\}_{n \in \omega},\left\{V_{n}\right\}_{n \in \omega}$ be sequences of closed neighborhoods of the points $x$ and $y$ in $Y$, respectively. Then there exist points $x^{\prime} \in X \cap \bigcap_{n \in \omega} U_{n}$ and $y^{\prime} \in X \cap \bigcap_{n \in \omega} V_{n}$ such that $x^{\prime} \leq y^{\prime}$.

Proof Replacing each set $U_{n}$ by $\bigcap_{i<n} U_{i}$, we can assume that $U_{n+1} \subseteq U_{n}$ for all $n \in \omega$. By the same reason, we can assume that the sequence $\left(V_{n}\right)_{n \in \omega}$ is decreasing. For every $n \in \omega$ denote by $U_{n}^{\circ}$ and $V_{n}^{\circ}$ the interiors of the sets $U_{n}$ and $V_{n}$ in $Y$.

By induction we shall construct sequences $\left(x_{n}\right)_{n \in \omega}$ and $\left(y_{n}\right)_{n \in \omega}$ of points of $X$ such that for every $n \in \omega$ the following conditions are satisfied:

$\left(1_{n}\right) x_{n} \leq y_{n}$

(2n) $\left\{x_{i} \cdots x_{n}, x_{i} \cdots x_{n} x\right\} \subseteq U_{i}^{\circ}$ for all $i \leq n$;

$\left(3_{n}\right)\left\{y_{i} \cdots y_{n}, y_{i} \cdots y_{n} y\right\} \subseteq V_{i}^{\circ}$ for all $i \leq n$.

To choose the initial points $x_{0}, y_{0}$, use the separate continuity of the semilattice operation and find neighborhoods $U_{0}^{\prime} \subseteq U_{0}^{\circ}$ and $V_{0}^{\prime} \subseteq V_{0}^{\circ}$ of $x$ and $y$ in $Y$ such that $U_{0}^{\prime} x \subseteq U_{0}^{\circ}$ and $V_{0}^{\prime} y \subseteq V_{0}^{\circ}$. By our assumption, there are points $x_{0} \in X \cap U_{0}^{\prime}$ and $y_{0} \in X \cap V_{0}^{\prime}$ such that $x_{0} \leq y_{0}$. The choice of the neighborhoods $U_{0}^{\prime}$ and $V_{0}^{\prime}$ ensures that the conditions $\left(2_{0}\right)$ and $\left(3_{0}\right)$ are satisfied.

Now assume that for some $n \in \mathbb{N}$ points $x_{0}, \ldots, x_{n-1}$ and $y_{0}, \ldots, y_{n-1}$ of $X$ are chosen so that the conditions $\left(1_{n-1}\right)-\left(3_{n-1}\right)$ are satisfied. The condition $\left(2_{n-1}\right)$ implies that for every $i \leq n$ we have the inclusion $x_{i} \cdots x_{n-1} x x=x_{i} \cdots x_{n-1} x \in U_{i}^{\circ}$ (if $i=n$, then we understand that $x_{i} \cdots x_{n-1} x=x$ ). Using the continuity of the shift $s_{x}: Y \rightarrow Y, s_{x}: z \mapsto x z$, we can find a neighborhood $U_{n}^{\prime} \subseteq Y$ of $x$ such that $x_{i} \cdots x_{n-1} \cdot\left(U_{n}^{\prime} \cup U_{n}^{\prime} x\right) \subseteq U_{i}^{\circ}$ for every $i \leq n$. By analogy, we can find a neighborhood $V_{n}^{\prime} \subseteq Y$ of $y$ such that $y_{i} \cdots y_{n-1} \cdot\left(V_{n}^{\prime} \cup V_{n}^{\prime} y\right) \subseteq V_{i}^{\circ}$ for every $i \leq n$. By our assumption, there are points $x_{n} \in X \cap U_{n}^{\prime}$ and $y_{n} \in X \cap V_{n}^{\prime}$ such that $x_{n} \leq y_{n}$. The choice of the neighborhoods $U_{0}^{\prime}$ and $V_{0}^{\prime}$ ensures that the conditions $\left(2_{n}\right)$ and $\left(3_{n}\right)$ are satisfied. This completes the inductive step. 
Now for every $i \in \omega$ consider the chain $C_{i}=\left\{x_{i} \cdots x_{n}: n \geq i\right\} \subseteq U_{i}^{\circ}$ in $X$. By the completeness of $X$, this chain has inf $C_{i} \in X \cap \overline{C_{i}} \subseteq X \cap \overline{U_{i}^{\circ}} \subseteq X \cap U_{i}$. Observing that inf $C_{i} \leq x_{i} x_{i+1} \cdots x_{n} \leq x_{i+1} \cdots x_{n}$ for all $i>n$, we see that inf $C_{i}$ is a lower bound of the chain $C_{i+1}$ and hence $\inf C_{i} \leq \inf C_{i+1}$. By the completeness of $X$, for every $i \in \omega$ the chain $D_{i}:=\left\{\inf C_{j}: j \geq i\right\} \subseteq U_{i}$ has $\sup D_{i} \in X \cap \overline{D_{i}} \subseteq X \cap U_{i}$. Since the sequence $\left(\inf C_{i}\right)_{i \in \omega}$ is increasing, we get $\sup D_{0}=\sup D_{i} \in X \cap U_{i}$ for all $i \in \omega$. Consequently, $\sup D_{0} \in X \cap \bigcap_{i \in \omega} U_{i}$.

By analogy, for every $k \in \omega$ consider the chain $E_{i}=\left\{y_{i} \cdots y_{n}: n \geq i\right\} \subseteq V_{i}^{\circ}$ in $X$. By the completeness of $X$, this chain has inf $E_{i} \in X \cap \overline{E_{i}} \subseteq X \cap \overline{V_{i}^{\circ}} \subseteq X \cap V_{i}$. By the completeness of $X$, for every $i \in \omega$ the chain $F_{i}:=\left\{\inf E_{j}: j \geq i\right\} \subseteq V_{i}$ has $\sup F_{i} \in X \cap \overline{F_{i}} \subseteq X \cap \overline{V_{i}}=X \cap V_{i}$. Since the sequence (inf $\left.E_{i}\right)_{i \in \omega}$ is increasing, we get $\sup F_{0}=\sup F_{i} \in X \cap V_{i}$ for all $i \in \omega$. Consequently, $\sup F_{0} \in X \cap \bigcap_{i \in \omega} V_{i}$.

To finish the proof of Lemma 2 , it suffices to show that $\sup D_{0} \leq \sup F_{0}$. The inductive conditions $\left(1_{n}\right), n \in \omega$, imply that $\inf C_{i} \leq \inf E_{i}$ for all $i \in \omega$ and $\sup D_{0}=\sup \left\{\inf C_{i}: i \in \omega\right\} \leq \sup \left\{\inf E_{i}: i \in \omega\right\}=\sup F_{0}$.

The following two lemmas imply Theorem 6.

Lemma 3 Let $Y$ be an $\omega$-Lawson semitopological semilattice. For any complete subsemilattice $X \subseteq Y$ the natural partial order $\leq_{X}$ of $X$ is closed in $Y \times Y$.

Proof By Corollary 3, the complete semitopological semilattice $X$ is both up-complete and down-complete. To show that the partial order $\leq_{X}:=\{(x, y) \in X \times X: x \leq y\}$ is closed in $Y \times Y$, take any pair $\left(y_{1}, y_{2}\right)$ in the closure of the set $\leq_{X}$ in $Y \times Y$. For every $i \in\{1,2\}$, let $\mathfrak{U}_{i}$ be the set of all countable families $\mathcal{U}$ of closed neighborhoods of $y_{i}$ in $Y$ such that $\bigcap \mathcal{U}$ is a subsemilattice of $Y$. By Lemma 2, for any $\mathcal{U}_{1} \in \mathfrak{U}_{1}$ and $\mathcal{U}_{2} \in \mathfrak{U}_{2}$ there are points $x_{1} \in X \cap \cap \mathcal{U}_{1}$ and $x_{2} \in X \cap \cap \mathcal{U}_{2}$ such that $x_{1} \leq x_{2}$. In particular, the closed subsemilattice $X \cap \cap \mathcal{U}_{1}$ is not empty and has the smallest element $\inf \left(X \cap \bigcap \mathcal{U}_{1}\right) \in X$ (by the down-completeness of $\left.X\right)$. Denote this smallest element by $x\left(\mathcal{U}_{1}\right)$. It follows that $x\left(\mathcal{U}_{1}\right):=\inf \left(X \cap \cap \mathcal{U}_{1}\right) \leq x_{1} \leq x_{2}$. Consequently, the closed subsemilattice $\left(\uparrow x\left(\mathcal{U}_{1}\right)\right) \cap\left(X \cap \bigcap \mathcal{U}_{2}\right) \ni x_{2}$ is not empty and has the smallest element (by down-completeness of $X$ ), which will be denoted by $y\left(\mathcal{U}_{1}, \mathcal{U}_{2}\right)$. Observe that $x\left(\mathcal{U}_{1}\right) \in X \cap \bigcap \mathcal{U}_{1}, y\left(\mathcal{U}_{1}, \mathcal{U}_{2}\right) \in X \cap \bigcap \mathcal{U}_{2}$ and $x\left(\mathcal{U}_{1}\right) \leq y\left(\mathcal{U}_{1}, \mathcal{U}_{2}\right)$. For any families $\mathcal{U}_{1} \in \mathfrak{U}_{1}$ and $\mathcal{U}_{2}, \mathcal{U}_{2}^{\prime} \in \mathfrak{U}_{2}$ with $\mathcal{U}_{2} \subseteq \mathcal{U}_{2}^{\prime}$ we have $y\left(\mathcal{U}_{1}, \mathcal{U}_{2}\right) \leq$ $y\left(\mathcal{U}_{1}, \mathcal{U}_{2}^{\prime}\right)$. Therefore, the set $\left\{y\left(\mathcal{U}_{1}, \mathcal{U}_{2}\right): \mathcal{U}_{2} \in \mathfrak{U}_{2}\right\} \subseteq X$ is up-directed and by the up-completeness of $X$, it has the smallest upper bound in $X$, which will be denoted by $y\left(\mathcal{U}_{1}\right)$. It follows that $x\left(\mathcal{U}_{1}\right) \leq y\left(\mathcal{U}_{1}\right)$. We claim that $y\left(\mathcal{U}_{1}\right)=y_{2}$. In the opposite case we can use the $\omega$-Lawson property of $Y$ and choose a countable family $\mathcal{U}_{2}^{\prime} \in \mathfrak{U}_{2}$ such that $y\left(\mathcal{U}_{1}\right) \notin \bigcap \mathcal{U}_{2}^{\prime}$. Taking into account that the set $\left\{y\left(\mathcal{U}_{1}, \mathcal{U}_{2} \cap \mathcal{U}_{2}^{\prime}\right): \mathcal{U}_{2} \in \mathfrak{U}_{2}\right\}$ is cofinal in $\left\{y\left(\mathcal{U}_{1}, \mathcal{U}_{2}\right): \mathcal{U}_{2} \in \mathfrak{U}_{2}\right\}$, we conclude that

$$
y\left(\mathcal{U}_{1}\right)=\sup \left\{y\left(\mathcal{U}_{1}, \mathcal{U}_{2}\right): \mathcal{U}_{2} \in \mathfrak{U}_{2}\right\}=\sup \left\{y\left(\mathcal{U}_{1}, \mathcal{U}_{2} \cap \mathcal{U}_{2}^{\prime}\right): \mathcal{U}_{2} \in \mathfrak{U}_{2}\right\} \in \bigcap \mathcal{U}_{2}^{\prime},
$$

which contradicts the choice of the family $\mathcal{U}_{2}^{\prime}$. This contradiction shows that $y_{2}=$ $y\left(\mathcal{U}_{1}\right) \in X$. Now we see that $x\left(\mathcal{U}_{1}\right) \leq y\left(\mathcal{U}_{1}\right)=y_{2}$ for every $\mathcal{U}_{1} \in \mathfrak{U}_{1}$. By the upcompleteness of the semitopological semilattice $X$, the up-directed subset $\left\{x\left(\mathcal{U}_{1}\right)\right.$ : 
$\left.\mathcal{U}_{1} \in \mathfrak{U}_{1}\right\}$ has the smallest upper bound $x \in X$. It follows from $x\left(\mathcal{U}_{1}\right) \leq y_{2}$ for all $\mathcal{U}_{1} \in \mathfrak{U}_{1}$ that $x \leq y_{2}$.

It remains to check that $x=y_{1}$. In the opposite case, using the $\omega$-Lawson property of $X$, we can find a countable family $\mathcal{U}_{1}^{\prime} \in \mathfrak{U}_{1}$ such that $x \notin \bigcap \mathcal{U}_{1}^{\prime}$. Taking into account that the family $\left\{x\left(\mathcal{U}_{1} \cap \mathcal{U}_{1}^{\prime}\right): \mathcal{U}_{1} \in \mathfrak{U}_{1}\right\}$ is cofinal in $\left\{x\left(\mathcal{U}_{1}\right): \mathcal{U}_{1} \in \mathfrak{U}_{1}\right\}$, we conclude that

$$
x=\sup \left\{x\left(\mathcal{U}_{1}\right): \mathcal{U}_{1} \in \mathfrak{U}_{1}\right\}=\sup \left\{x\left(\mathcal{U}_{1} \cap \mathcal{U}_{1}^{\prime}\right): \mathcal{U}_{1} \in \mathfrak{U}_{1}\right\} \in \bigcap \mathcal{U}_{1}^{\prime}
$$

which contradicts the choice of $\mathcal{U}_{1}^{\prime}$. This contradiction shows that $y_{1}=x \in X$. Therefore we obtain that $\left(y_{1}, y_{2}\right) \in X \times X$ and $y_{1}=x \leq y_{2}$, which means that $\left(y_{1}, y_{2}\right) \in \leq X$.

Lemma 4 Each complete subsemilattice $X$ of an $\omega$-Lawson semitopological semilattice $Y$ is closed in $Y$.

Proof By Lemma 3, the partial order $\leq_{X}:=\{(x, y) \in X \times X: x y=x\}$ is a closed subset of $Y \times Y$. By Corollary 3, the complete semilattice $X$ has the smallest element $\min X \in X$. Consider the continuous map $f: Y \rightarrow Y \times Y, f: y \mapsto(\min X, y)$, and observe that $X=f^{-1}\left(\leq_{X}\right)$ is a closed subset of $X$, being the preimage of the closed set $\leq_{X}$ under the continuous map $f$.

Finally, we prove Corollary 1.

Lemma 5 For every continuous homomorphism $h: X \rightarrow Y$ from a complete topologized semilattice $X$ to an $\omega$-Lawson semitopological semilattice $Y$, the image $h[X]$ is closed in $Y$.

Proof Observe that the $\omega$-Lawson property of $Y$ implies that the semitopological semilattice $Y$ is Hausdorff and hence $\uparrow$-closed. By Lemma 1, the semitopological semilattice $h[X]$ of $Y$ is complete and by Lemma $4, h[X]$ is closed in $Y$.

Open Access This article is licensed under a Creative Commons Attribution 4.0 International License, which permits use, sharing, adaptation, distribution and reproduction in any medium or format, as long as you give appropriate credit to the original author(s) and the source, provide a link to the Creative Commons licence, and indicate if changes were made. The images or other third party material in this article are included in the article's Creative Commons licence, unless indicated otherwise in a credit line to the material. If material is not included in the article's Creative Commons licence and your intended use is not permitted by statutory regulation or exceeds the permitted use, you will need to obtain permission directly from the copyright holder. To view a copy of this licence, visit http://creativecommons.org/licenses/by/4.0/.

\section{References}

1. Banakh, T., Bardyla, S.: Characterizing chain-finite and chain-compact topological semilattices. Semigroup Forum 98(2), 234-250 (2019)

2. Banakh, T., Bardyla, S.: The interplay between weak topologies on topological semilattices. Topol. Appl. 259, 134-154 (2019) 
3. Banakh, T., Bardyla, S.: Completeness and absolute $H$-closedness of topological semilattices. Topol. Appl. 260, 189-202 (2019)

4. Banakh, T., Bardyla, S.: On images of complete subsemilattices in sequential semitopological semilattices. Semigroup Forum 100, 662-670 (2020)

5. Banakh, T., Bardyla, S.: Complete topologized posets and semilattices. Topol. Proc. 57, 177-196 (2021)

6. Banakh, T., Bardyla, S., Ravsky, A.: The closedness of complete subsemilattices in functionally Hausdorff semitopological semilattices. Topol. Appl. 267, 106874 (2019)

7. Banakh, T., Bardyla, S., Ravsky, A.: A metrizable semitopological semilattice with non-closed partial order. Top. Algebra Appl. 8(1), 67-75 (2020)

8. Banakh, T., Bardyla, S., Ravsky, A.: A metrizable Lawson semitopological semilattice with non-closed partial order. Proc. Intern. Geom. Center 13(3), 10-17 (2020)

9. Bruns, G.: A lemma on directed sets and chains. Arch. Math. 18(6), 561-563 (1967)

10. Carruth, J.H., Hildebrant, J.A., Koch, R.J.: The Theory of Topological Semigroups, vol. II. Marcel Dekker Inc, New York and Basel (1986)

11. Gutik, O., Repovš, D.: On linearly ordered $H$-closed topological semilattices. Semigroup Forum 77(3), 474-481 (2008)

12. Iwamura, T.: A lemma on directed sets. Zenkoku Shijo Sugaku Danwakai 262, 107-111 (1944)

13. Lawson, J.D.: Topological semilattices with small semilattices. J. London Math. Soc. (2) 1(1), 719-724 (1969)

14. Lawson, J.D.: Joint continuity in semitopological semigroups. Illinois J. Math. 18(2), 275-285 (1974)

15. Markowsky, G.: Chain-complete posets and directed sets with applications. Algebra Univers. 6(1), 53-68 (1976)

16. Stepp, J.W.: Algebraic maximal semilattices. Pac. J. Math. 58(1), 243-248 (1975)

Publisher's Note Springer Nature remains neutral with regard to jurisdictional claims in published maps and institutional affiliations. 